\title{
Advancement on Clinical Application of mTOR Inhibitors in Gastrointestinal Cancers
}

\author{
Yingqi Feng*, Yongshou Chen*, Yixin Ren*, Shuang Cao\#, Heng Zhang\# \\ Key Laboratory for Green Chemical Process of Ministry of Education, School of Chemical Engineering and Pharmacy, Wuhan \\ Institute of Technology, Wuhan, China \\ Email: "zhzpthm@163.com, " caoshuangknight@163.com
}

How to cite this paper: Feng, Y.Q., Chen, Y.S., Ren, Y.X., Cao, S. and Zhang, H. (2020) Advancement on Clinical Application of mTOR Inhibitors in Gastrointestinal Cancers. Journal of Biosciences and Medicines, 8, 45-57.

https://doi.org/10.4236/jbm.2020.84004

Received: March 2, 2020

Accepted: April 7, 2020

Published: April 10, 2020

Copyright $\odot 2020$ by author(s) and Scientific Research Publishing Inc. This work is licensed under the Creative Commons Attribution International License (CC BY 4.0).

http://creativecommons.org/licenses/by/4.0/

\begin{abstract}
Gastrointestinal cancers, including esophageal cancer, gastric cancer, liver cancer, pancreatic cancer, colorectal cancer, are serious threats to people's health, and traditional therapeutic drugs have limited effect on them. Signaling pathway related to mammalian target of rapamycin (mTOR) plays a very important role either in the germination or development of multiple gastrointestinal cancers. Inhibiting mTOR can effectively impede the development of tumor and improve the sensitivity of tumor to radiotherapy or chemotherapy. The research on the application of mTOR inhibitors in gastrointestinal cancers increases with years. A lot of operational schemes independent or combined with others are being explored, some of which are worthy of attention. This paper summarizes the data of all published clinical trials and reviews the clinical application of mTOR inhibitors in the treatment of gastrointestinal cancers in recent years.
\end{abstract}

\section{Keywords \\ mTOR, Gastrointestinal Cancers, Targeted Drug, Sensitization}

\section{Introduction}

Gastrointestinal cancers, including esophageal carcinoma, gastric carcinoma, hepatocellular carcinoma, pancreatic cancer and colorectal cancer, are the main type of cancer. In 2019, there will be 290,000 cases of gastrointestinal cancers occurred in the USA, and more than 170,000 people will be killed by these diseases [1]. Among them, colorectal cancer has the highest incidence rate, and liver cancer has the highest mortality rate. Although many patients have received

*These authors contributed equally to this work.

${ }^{\#}$ Corresponding authors. 
corresponding treatment in the early stage, most of them are still irreversibly deteriorating. Nowadays, the first choice to deal with advanced or recurrent gastrointestinal cancers is surgery or platinum drugs, and the molecularly targeted drugs can be used for patients with platinum resistance [2]. Since more than ten years ago, anti-angiogenic therapy with molecular targeted drugs has been introduced into the treatment of advanced gastrointestinal cancers, among which the phosphatidylinositol 3-kinase (PI3K)/mTOR inhibitors, epidermal growth factor receptor (EGFR) inhibitors, fibroblast growth factor receptor (FGFR) inhibitors and c-Met inhibitors are the representative drugs [3]. mTOR inhibitors are potential targeted antitumor agents with high activity and low toxicity, and their applications in the treatment of gastrointestinal cancer are of particular concern [4] [5]. This paper summarized the clinical application of mTOR inhibitors in gastrointestinal cancer, and provided reference for the related researchers.

\section{2. mTOR and mTOR Inhibitors}

mTOR is the mammalian target of rapamycin composed of 2549 amino acids with a molecular weight of $289 \mathrm{kDa}$, belonging to serine/threonine protein kinase. It widely exists in various mammalian cells and participates in many cell processes, such as cell growth, proliferation, protein synthesis, transcription and metabolism [6]. From the C-terminal to the $\mathrm{N}$-terminal, mTOR is successively distributed with focal adhesion targeting domain of C-terminal, negative regulatory domain, kinase domain, FKBP12 rapamycin binding and focal adhesion targeting domain, and it belongs to phosphoinositide 3-kinase-related protein kinases (PIKKs) [7]. mTOR can form two different complexes, mTORC1 and mTORC2 by combining with different proteins. The upstream activators and downstream effectors of mTORC1 and mTORC2 are different [8], and they coordinate each other to regulate the cell cycle process [6].

In the PI3K/Akt/mTOR signaling pathway (Figure 1), the transformation from phosphorylinositol $(4,5)$ bisphosphonate (PIP2) into phosphorylinositol $(3,4,5)$ trisphosphonate (PIP3) will be promoted after activating phosphorylinositol-3 kinase (PI3K), and then the phosphorylinoside-dependent kinase-1 (PDK-1) is activated. PDK-1 and PDK-2 work together to phosphorylate threonine at 308 site as well as serine at 473 site of protein kinase B (Akt), thus activating Akt and regulating mTORC1. The abnormal signal of this pathway can directly lead to cell proliferation or even cancer. For example, PTEN can inhibit the transformation from PIP2 to PIP3, thus regulating the activation of Akt. Therefore, when PTEN function is lost, Akt and mTOR in downstream will be activated abnormally. A number of studies have shown that mutations in PTEN or PI3K are directly related to endometrial cancer, cervical cancer or ovarian cancer [9].

The phosphorylation of eukaryotic translation initiation factor $4 \mathrm{E}$-binding protein 1 (4EBP1) in tumor cells can be inhibited by mTOR inhibitors, thus the expression of growth protein is reduced and tumor cells will be stopped in G1 


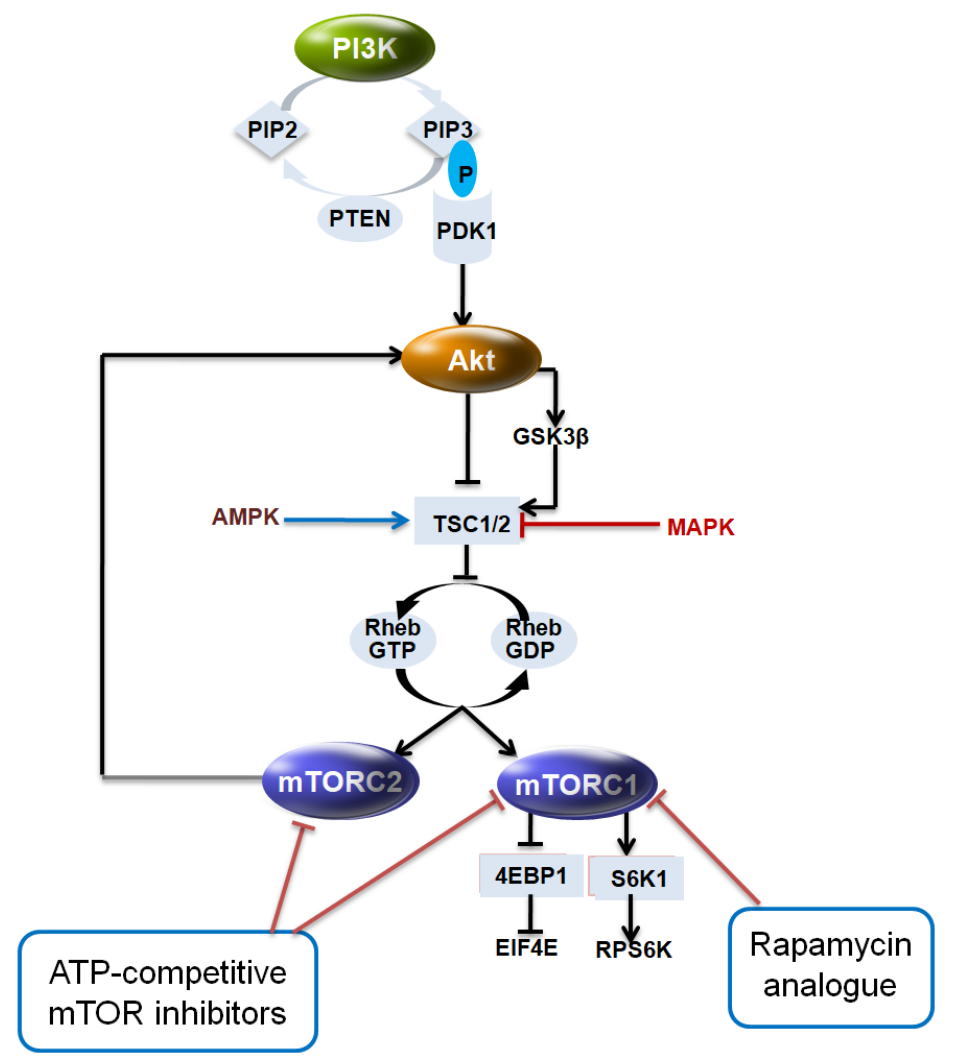

Figure 1. mTOR signaling pathways and action principle of mTOR inhibitors. PTEN: phosphatase and tensin homolog deleted on chromosome ten; AMPK: Adenosine 5'-monophosphate (AMP)-activated protein kinase; MAPK: Mitogen-activated protein kinase; 4EBP1: Products the eukaryotic initiation factor 4E binding protein 1; S6K1: S6 kinase 1; EIF4E: Eukaryotic initiation factor 4E; RPS6K: Recombinant Ribosomal Protein S6 Kinase Beta 1.

phase, or even finally die off. In addition, it has been found in recent years that mTOR inhibitors can also enhance the sensitivity of tumor cells to radiotherapy [10] [11] or chemotherapy [12]. These mechanisms make mTOR inhibitors becoming antitumor targeted drugs with highly effective and low toxic.

The reported mTOR inhibitors can be divided into two categories, the one is rapamycin derivatives acting on FRB domain, and the other one is ATP competitive mTOR inhibitors acting on protein kinase domain. Rapamycin (sirolimus), everolimus, ridaforolimus, temsirolimus (CCI-779) [13] belong to rapamycin derivatives, which have been approved for marketing. Rapamycin is the first mTOR inhibitor and the first one used in clinic. It can inhibit the growth of breast cancer, ovarian cancer, prostate cancer, renal cancer, cervical cancer and other tumor cells in a concentration dependent manner. Rapamycin opens a new way for cancer treatment, but the poor water solubility and low bioavailability $(<15 \%)$ limit its application. In order to overcome the shortcomings of rapamycin in physical and chemical properties, its derivatives, everolimus, ridaforolimus, CCI-779, were developed one after another. ATP competitive mTOR inhi- 
bitors are a new class of compounds developed in recent years, which act on the ATP binding cavity of mTOR and have excellent activity. However, due to toxicity and the shortcomings of pharmacokinetics, all of them are sluggish in stage of clinical research. For instance, there are 20 clinical trials about mTOR inhibitor BEZ235 registered in www.clinicaltrials.gov, but all of them are terminated in phase I/II. The representative compounds of ATP competitive mTOR inhibitors are BEZ-235 [14], Wye-125132 [15], Torin2 [16], PF-04691502 [17], etc.

\section{The Application of mTOR Inhibitors in Treating Gastrointestinal Cancers}

mTOR inhibitors have been used in treating gastrointestinal cancers for about 10 years, the therapeutic schemes and clinical results are different according to different cancers. In this paper, the clinical trials database collected by the National Medical Library of the USA was investigated and five main types of gastrointestinal cancers were searched respectively. The published clinical trial data and results were summarized below.

\subsection{Esophagus Carcinoma}

The incidence rate of esophagus carcinoma ranks eighteenth in all cancers, and the five year survival rate is about $19.9 \%$. In 2019 , there will be 17,650 new cases of esophagus carcinoma in the USA, accounting for $1.0 \%$ of new cancer cases, and 16,080 patients will be killed by this cancer accounting for $2.6 \%$ of all cancer deaths [1]. The PI3K/mTOR pathway is altered in many cancers, and several inhibitors of this pathway are already approved for a variety of malignancies, however there are few reports on the application of mTOR inhibitors in the treatment of esophageal cancer [18].

In 2009, Translational Oncology Research International in Unite State launched phase II clinical study numbered NCT00985192 [18] to evaluate the efficacy and safety of everolimus in previously treated patients with metastatic adenocarcinoma of the esophagus. The median age of the 45 volunteers recruited for the trial was 64 years old. Patients with advanced esophagus carcinoma who progressed after 1 - 2 prior regions received oral everolimus $10 \mathrm{mg}$ daily. The most common grade 3 - 4 adverse events in the trial were fatigue (24\%) and thrombocytopenia (22\%). The results showed that one patient had a partial response and $39 \%$ patients had stable disease. Median overall survival (OS) was 3.4 months, and Progression Free Survival (PFS) was 1.8 months. The results of this study did not show the significant effect of everolimus in the treatment of esophagus carcinoma. However, a strong correlation between the severity of esophageal cancer and the diagnostic factor PS6 has been found.

\subsection{Gastric Carcinoma}

The incidence rate of gastric carcinoma (GC) ranks fifteenth among all cancers, and the five year survival rate is $31.5 \%$. In 2019 , there will be 27,510 new cases of 
GC in the USA, accounting for $1.6 \%$ of new cancer cases, and 11140 patients will die accounting for $1.8 \%$ of all cancer deaths [1]. Surgical operation may be effective in early treatment of GC, but inoperable for about two-thirds patients. New targeted therapies for GC have emerged based on many cancer-related molecular pathways. In human GC, PI3K/Akt and mTOR are known to be activated in approximately $30 \%$ and $60 \%$ of patients, respectively. Therefore, in the era of molecular-targeted agents, inhibition of the mTOR pathway represents a novel therapeutic strategy in the treatment of GC [19].

In 2007, Novartis Pharmaceuticals launched a single arm, multi-center phase II study (NCT00519324) [20] of everolimus in patients with advanced GC. This study was to evaluate the efficacy and safety of everolimus in the treatment of advanced GC. A total of 54 patients with median age of 63 years old were enrolled in the trial, and given $10 \mathrm{mg} /$ day of everolimus orally. The results showed that the tumor volume of $45 \%$ patients was smaller than baseline. The disease control rate (DCR) was $56.0 \%$, and the median progression-free survival (PFS) was 2.7 months. In the follow-up time of median (9.6 months), the median OS was 10.1 months. Common grade 3 or 4 adverse events included anemia, hyponatremia, increased $\gamma$-glutamyltransferase, and lymphopenia. Grade 1 or 2 pneumonitis was reported in eight patients (15.1\%).

In 2008, National Taiwan University Hospital launched a phase II study (NCT00632268) [21]. This study used low-dose everolimus plus cisplatin and HDFL (High-dose 5-Fluorouracil and Leucovorin) chemotherapy for first-line treatment of unresectable, recurrent or metastatic GC. The trial enrolled 40 patients including 19 men and 21 women with the median age of 54.1. These eligible chemotherapy-naïve patients with advanced GC received low dose of everolimus (10 mg/day) plus cisplatin and high dose of DHFL (cisplatin $35 \mathrm{mg} / \mathrm{m}^{2}$ intravenous infection for 24 hours on days 1 and 8, 5-fluororouracil $2000 \mathrm{mg} / \mathrm{m}^{2}$ and leucovorin $300 \mathrm{mg} / \mathrm{m}^{2}$ intravenous infection for 24 hours on days 1, 8 and 15) 24 hours a week in 28 days. The primary end point was objective response rate (ORR). The results showed that the ORR was 52.5\% (21 confirmed partial response). Median PFS and total survival were 6.9 and 10.5 months, respectively. Most of the adverse events were mild. The results showed that adding low-dose everolimus to cisplatin-HDFL chemotherapy could not increase ORR as planned, but prolong the PFS of patients with advanced GC.

In 2008, Asan Medical Center launched a phase II study numbered NCT00729482 [22] to evaluate the activity and safety of everolimus and identify potential biomarkers in patients with advanced GC, who failed both fluoropyrimidine and platinum. A total of 54 patients were enrolled in the trial, taking everolimus 10 $\mathrm{mg}$ /day orally. The results of the trial showed that 2 patients $(3.7 \%)$ are partially alleviated, and DCR was 38.9\%. Median PFS was 1.7 months and median OS was 8.3 months. The main toxicity was grade 1 or 2 athenia $(96.3 \%)$, and the most common grade 3 or 4 non-hematologic toxicity was hyperglycemia (20.4\%). Three patients gained grade 3 or 4 pneumonia. Notably, there were two treat- 
ment-related deaths. The test shows that everolimus is active against a limited number of patients with advanced GC. At the same time, the test also proved that pS6ser240/4 might be a potential predictive biological index in patients with AGC.

In 2009, Samsung Medical Center launched a phase I/II trial of everolimus/capecitabine in refractory GC (NCT01099527) [23]. This study was to assess the efficacy and safety of combination regimen of capecitabine plus everolimus in patients with refractory gastric cancer who have failed to at least two cytotoxic regimens. The 47 eligible patients recruited in the trial were treated with capecitabine $650 \mathrm{mg} / \mathrm{m}^{2}$ twice daily and everolimus $5 \mathrm{mg}$ twice daily every 3 weeks until disease progression or unacceptable toxicity. The primary endpoint of the study was overall response (partial or complete response) and the secondary endpoints were PFS (time between registration and disease progression or death) and OS. The results showed that in 43 cases of evaluable response, partial response occurred in 5 patients, 18 patients had stable disease, ORR was 10.6\%, and disease control rate was $48.9 \%$. Median PFS and total survival were 11 weeks and 21 weeks, respectively. The grade 3 side effects, such as nausea, diarrhea and stomatitis, occurred in 2 cases, 3 cases and 3 cases respectively. Elevated liver enzymes arose in 21 patients, no pulmonary fibrosis appeared. This study showed that treatment with capecitabine $650 \mathrm{mg} / \mathrm{m}^{2}$ twice a day and everolimus $5 \mathrm{mg}$ twice a day were effective for a few patients with more seriously GC who had been treated in the early stage.

Owning to the good effect of oral everolimus in the phase II study before the treatment of advanced GC, Novartis pharmaceuticals launched a randomized, double blind, multi-center phase III study in 2009 (NCT00879333) [24]. This study was to assess the efficacy and safety of patients with AGC after progression on 1 or 2 prior systemic chemotherapy using everolimus. The trial enrolled 656 patients, wherein the median age is 62.0 , and of whom $73.6 \%$ is men. Median OS of everolimus and placebo groups was 5.4 months and 4.3 months, respectively. Median PFS was 1.7 months with everolimus and 1.4 months with placebo. Common grade 3 or 4 adverse events include anemia, loss of appetite, and fatigue, and there is no significant difference in the security of various groups. Compared with the best supportive care (BSC), everolimus did not significantly improve the OS of patients with AGC. The safety of everolimus in the treatment of GC is consistent with that in other cancers.

In the clinical trials of mTOR inhibitors, GC has the most records, which shows the researchers' attention in this field.

\subsection{Hepatocellular Carcinoma}

The incidence rate of hepatocellular carcinoma (HCC) is thirteenth among all cancers, with a five year survival rate of $18.4 \%$. In 2019, 42,030 new cases of HCC will arise in the USA, accounting for $2.4 \%$ of new cancer cases, and 31,780 patients will be killed by this cancer, accounting for $5.2 \%$ of all cancer deaths [1]. 
Because the occurrence of liver cancer is relatively unconspicuous, it is often in late stage when diagnosed, and only about $30 \%$ patients can be treated by surgery [25]. Studies have shown that the mTOR/AKT pathway is frequently up-regulated in $40 \%-50 \%$ of hepatocellular carcinomas. Inhibition of AKT and mTOR signals not only inhibits the production of VEGF, but also regulates the expression of other angiogenic factors (such as nitric oxide and angiogenin), thereby effectively delaying the progression of HCC [26]. In terms of HCC cells, mTOR can also enhance the resistance of HCC to chemotherapy by transforming ordinary HCC cells into tumor stem cells. Overactivated mTOR can change the biological behavior and characteristics of HCC cells, so that some HCC cells have characteristics similar to those of stem cells and form cancer stem cells (CSC) [27]. After transformation into tumor stem cells, the HCC cells obtained more significant invasion and migration capacity, and showed stronger tolerance to anti-tumor drugs such as sorafenib [28].

In 2009, University of Regensburg launched an open label, randomized, and multi-center phase III study (NCT00355862) [29] in order to compare sirolimus-containing versus sirolimus-free immunosuppression in patients undergoing liver transplantation for HCC. A total of 525 patients were recruited and divided into two groups: group A (264 patients) did not receive sirolimus, group B (261 patients) received sirolimus. The primary end point was relapse free survival (RFs), and OS was the secondary end point. The results showed that the RFs rate of group A was $64.5 \%$, and that of group B was $70.2 \%$, which had no statistical difference. Group B showed a better RFs rate in three years after transplantation, and a better OS in five years. Interestingly, low-risk patients benefit the most from sirolimus rather than high-risk patients. The number of serious adverse events occurred in group A (860 cases) and group B (874 cases) was same. The results showed that the RFs of liver cancer patients treated with sirolimus had no significant improvement after 5 years. However, the benefits in RFs and OS were evident in the first five years, especially in low-risk patients. This trial provides the first high-level evidence for selecting immunosuppression in liver transplantation recipients with HCC.

It has been proved that mTOR inhibitors used directly in the treatment of HCC cannot work well, and may be more suitable as chemosensitizers and radiosensitizers [10] [30] for the treatment of HCC.

\subsection{Pancreatic Carcinoma}

The incidence rate of pancreatic carcinoma is eleventh in all cancers, with a five year survival rate of $9.3 \%$. In 2019, it is estimated that 56,770 new cases of pancreatic cancer will be added in the USA, accounting for $3.2 \%$ of the new cancer cases, and 45,750 patients will be killed by this cancer, accounting for $7.5 \%$ of the total cancer deaths [1]. Pancreatic carcinoma responds poorly to most chemotherapeutic drugs. As targeted therapies produce mild adverse reactions due to their specificity and targeted inhibition, they have become the preferred treatments for cancers in recent years [31]. 
In 2009, Novartis pharmaceuticals launched a randomized, double-blind, multi-center clinical phase II study (NCT01658436) to evaluate the efficacy and safety of everolimu in the treatment of advanced pancreatic neuroendocrine tumors (PNET). A total of 256 patients were enrolled in the clinical trial, 130 of whom were assigned to the everolimus group and 126 to the placebo group, with a median follow-up period of 43 months. The median PFS was 11 months in the everolimus group and 4.6 months in the placebo group. The most common side effects in the everolimus group were stomatitis, rash, diarrhea, tiredness, edema, abdominal pain, nausea, fever and headache. In view of the efficacy and unique mechanism, FDA approved Novartis pharmaceuticals' new drug Afinitor (everolimus) in May 2011 for treatment of PNET which cannot be surgically removed. The drug is the only one approved for PNET in the USA for nearly 30 years.

ATP competitive mTOR inhibitors have novel structures and inhibiting mechanisms, the representative one is BEZ235. In 2012, Novartis pharmaceuticals launched a multi-center, two stages, phase II study (NCT02315625) [32] to evaluate the efficacy of oral BEZ235 to patients with PNET. In the first group, 11 patients were given $400 \mathrm{mg}$ BEZ235 twice a day. Due to tolerance problems, another 20 patients received $300 \mathrm{mg}$ BEZ235 twice daily in the second group. The trial ended in June 2014, wherein 8 cases (72.7\%) in first group and 8 cases (40.0\%) in second group of grade 3 or 4 adverse events were reported, including hyperglycemia, diarrhea, nausea and vomiting. The trial showed that PNET patients with everolimus resistant had poor tolerance to BEZ235 at doses of 400 or $300 \mathrm{mg}$. Although evidence for disease stability was observed in the trial, the study did not enter the second phase due to poor patient tolerance.

\subsection{Colorectal Cancer}

The incidence rate of colorectal cancer is the fourth in all cancers, with a five year survival rate of $64.4 \%$. In 2019, it is estimated that 145,600 new cases of colorectal cancer will arise in the USA, accounting for $8.3 \%$ of new cancer cases, and 51,020 people will be killed by this cancer, accounting for $8.4 \%$ of all cancer deaths [1]. Because of the limited effect of mTOR inhibitors on colorectal cancer, all reported trials used them in combination with other drugs [33].

Up to now, the FDA has approved three kinds of anti-VEGF treatment methods for colorectal cancer: bevacizumab, ziv-aflibercept and regorafenib. Among them, bevacizumab is the most widely studied, and has shown significant efficacy in the treatment of colorectal cancer. mTOR can theoretically enhance the antitumor effect of anti-VEGF drugs. In vitro experiment, the combination of anti-VEGF and mTOR inhibitors showed better effects than using them independently. So, Novartis pharmaceuticals conducted a phase II study to test the efficacy of bevacizumab combined with everolimus in patients with refractory colorectal cancer.

In 2007, Novartis Pharmaceuticals and Genentech, Inc. launched a phase II study (NCT00597506) [34], and evaluate the efficacy of the combination of be- 
vacizumab and everolimus to refractory metastatic colorectal cancer (MCRC). A total of 50 patients were recruited for the trial and given bevacizumab (10 $\mathrm{mg} / \mathrm{kg}$ ) combined with everolimus (10 $\mathrm{mg} / \mathrm{day})$, then the biomarkers of the treatment were evaluated. The baseline biomarker profile of each patient was used as his/her reference control. A total of 41 biomarkers in every patient were analyzed. Three markers (FGFb, IL8, VEGF-C) were excluded from the statistical analysis, and more than $10 \%$ samples could not be detected. The changes of 26 markers were statistically significant $(\mathrm{P}<0.05)$, of which 12 markers were highly significant $(\mathrm{P}<0.0001)$. Among them, three markers including Ang2, MCP-1 and VEGF-R2 were down regulated, while MMP-2, PAI-1 activity, PAI-1 total level, PLGF, SDF-1, VCAM-1, VEGF-A, VEGF-D and vWF were up regulated. In this study, more than $20 \%$ patients had a stable period of disease for six months or more, which indicated that some patients benefited greatly from this combination therapy. Note that most of the patients who participated in this trial were previously resistant to bevacizumab treatment, indicating the addition of everolimus delayed the bevacizumab resistance and improved clinical results.

\section{Summary}

In recent years, drug-development in treatment of gastrointestinal tumors is relatively slow. mTOR inhibitors have a good application prospect due to its high bioactivity and low toxicity. Only $30 \%$ of clinical trial results about mTOR inhibitors have been published, with about 35\% of trials aborted for various reasons. According to the published clinical data, the effect of using mTOR inhibitor alone in treatment of gastrointestinal tumor is limited. This may be because the complex feedback regulation pathways in the tumor interfere with the therapeutic effect of mTOR inhibitors. Therefore, the best application direction of mTOR inhibitors in the future should be combination drug therapy (with platinum drugs, cytotoxic drugs, antibody drugs, radiotherapy, etc.). In addition, the expression of S6K1, an important factor in mTOR pathway, is correlated with tumor development, which can be used as a diagnostic factor for tumor development.

\section{Acknowledgements}

We gratefully acknowledge financial support from the program for Graduate Innovative Fund of Wuhan Institute of Technology (No. CX2018001) and Research Fund of Wuhan Institute of Technology (No. K201756 and No. 2019018).

\section{Conflicts of Interest}

The authors declare no conflicts of interest regarding the publication of this paper.

\section{References}

[1] National Cancer Institute. Cancer Statistics. 
https://seer.cancer.gov/statfacts/html/corp.html

[2] Zhao, Y., Zhao, Q., Kaboli, P.J., Shen, J., Li, M., Wu, X., Yin, J., Zhang, H., Wu, Y., Lin, L., Zhang, L., Wan, L., Wen, Q., Li, X., Cho, C.H., Yi, T., Li, J. and Xiao, Z. (2019) m1A Regulated Genes Modulate PI3K/AKT/mTOR and ErbB Pathways in Gastrointestinal Cancer. Translational Oncology, 12, 1323-1333.

https://doi.org/10.1016/j.tranon.2019.06.007

[3] Freis, P., Bollard, J., Lebeau, J., Massoma, P., Fauvre, J., Vercherat, C., Walter, T., Manie, S., Roche, C., Scoazec, J.Y. and Ferraro-Peyret, C. (2017) mTOR Inhibitors Activate PERK Signaling and Favor Viability of Gastrointestinal Neuroendocrine Cell Lines. Oncotarget, 8, 20974-20987. https://doi.org/10.18632/oncotarget.15469

[4] Li, J.C., Zhu, H.Y., Chen, T.X., Zou, L.Y., Wang, X.Y., Zhao, H.C. and Xu, J. (2013) Roles of mTOR and p-mTOR in Gastrointestinal Stromal Tumors. Asian Pacific Journal of Cancer Prevention, 14, 5925-5928.

https://doi.org/10.7314/APJCP.2013.14.10.5925

[5] Malley, C.O. and Pidgeon, G.P. (2016) The mTOR Pathway in Obesity Driven Gastrointestinal Cancers: Potential Targets and Clinical Trials. BBA Clinical, 5, 29-40. https://doi.org/10.1016/j.bbacli.2015.11.003

[6] Cao, S., Cao, R., Liu, X., Luo, X. and Zhong, W. (2016) Design, Synthesis and Biological Evaluation of Novel Benzothiazole Derivatives as Selective PI3Kbeta Inhibitors. Molecules, 21, 876-890. https://doi.org/10.3390/molecules21070876

[7] Yang, H., Rudge, D.G., Koos, J.D., Vaidialingam, B., Yang, H.J. and Pavletich, N.P. (2013) mTOR Kinase Structure, Mechanism and Regulation. Nature, 497, 217-223. https://doi.org/10.1038/nature12122

[8] Hay, N. and Sonenberg, N. (2004) Upstream and Downstream of mTOR. Genes \& Development, 18, 1926-1945. https://doi.org/10.1101/gad.1212704

[9] Bertelsen, B.I., Steine, S.J., Sandvei, R., Molven, A. and Laerum, O.D. (2006) Molecular Analysis of the PI3K-AKT Pathway in Uterine Cervical Neoplasia: Frequent PIK3CA Amplification and AKT Phosphorylation. International Journal of Cancer, 118, 1877-1883. https://doi.org/10.1002/ijc.21461

[10] Kahn, J., Hayman, T.J., Jamal, M., Rath, B.H., Kramp, T., Camphausen, K. and Tofilon, P.J. (2014) The mTORC1/mTORC2 Inhibitor AZD2014 Enhances the Radiosensitivity of Glioblastoma Stem-Like Cells. Neuro-Oncology, 16, 29-37. https://doi.org/10.1093/neuonc/not139

[11] Hayman, T.J., Wahba, A., Rath, B.H., Bae, H., Kramp, T., Shankavaram, U.T., Camphausen, K. and Tofilon, P.J. (2014) The ATP-Competitive mTOR Inhibitor INK128 Enhances in Vitro and in Vivo Radiosensitivity of Pancreatic Carcinoma Cells. Clinical Cancer Research, 20, 110-119.

https://doi.org/10.1158/1078-0432.CCR-13-2136

[12] Li, A., Zhang, R., Zhang, Y., Liu, X., Wang, R., Liu, J., Liu, X., Xie, Y., Cao, W., Xu, R., Ma, Y., Cai, W., Wu, B., Cai, S. and Tang, X. (2019) BEZ235 Increases Sorafenib Inhibition of Hepatocellular Carcinoma Cells by Suppressing the PI3K/AKT/mTOR Pathway. American Journal of Translational Research, 11, 5573-5585.

[13] Li, S., Liang, Y., Wu, M., Wang, X., Fu, H., Chen, Y. and Wang, Z. (2013) The Novel mTOR Inhibitor CCI-779 (Temsirolimus) Induces Antiproliferative Effects through Inhibition of mTOR in Bel-7402 Liver Cancer Cells. Cancer Cell International, 13, 30. https://doi.org/10.1186/1475-2867-13-30

[14] Serova, M., de Gramont, A., Tijeras-Raballand, A., Dos Santos, C., Riveiro, M.E., Slimane, K., Faivre, S. and Raymond, E. (2013) Benchmarking Effects of mTOR, PI3K, and Dual PI3K/mTOR Inhibitors in Hepatocellular and Renal Cell Carcino- 
ma Models Developing Resistance to Sunitinib and Sorafenib. Cancer Chemotherapy and Pharmacology, 71, 1297-1307. https://doi.org/10.1007/s00280-013-2129-6

[15] Yu, K., Shi, C., Toral-Barza, L., Lucas, J., Shor, B., Kim, J.E., Zhang, W.-G., Mahoney, R., Gaydos, C., Tardio, L., Kim, S.K., Conant, R., Curran, K., Kaplan, J., Verheijen, J., Ayral-Kaloustian, S., Mansour, T.S., Abraham, R.T., Zask, A. and Gibbons, J.J. (2010) Beyond Rapalog Therapy: Preclinical Pharmacology and Antitumor Activity of WYE-125132, an ATP-Competitive and Specific Inhibitor of mTORC1 and mTORC2. Cancer Research, 70, 621-631.

https://doi.org/10.1158/0008-5472.CAN-09-2340

[16] Luo, J., Pi, G., Xiao, H., Ye, Y., Li, Q., Zhao, L., Huang, H., Luo, H., Zhang, Q., Wang, D. and Wang, G. (2018) Torin2 Enhances the Radiosensitivity of MCF7 Breast Cancer Cells by Down-Regulating the mTOR Signaling Pathway and ATM Phosphorylation. Molecular Medicine Reports, 17, 366-373.

https://doi.org/10.3892/mmr.2017.7848

[17] Tonlaar, N., Galoforo, S., Thibodeau, B.J., Ahmed, S., Wilson, T.G., Yumpo Cardenas, P., Marples, B. and Wilson, G.D. (2017) Antitumor Activity of the Dual PI3K/MTOR Inhibitor, PF-04691502, in Combination with Radiation in Head and Neck Cancer. Radiotherapy and Oncology, 124, 504-512. https://doi.org/10.1016/j.radonc.2017.08.001

[18] Wainberg, Z.A., Soares, H.P., Patel, R., DiCarlo, B., Park, D.J., Liem, A., Wang, H.J., Yonemoto, L., Martinez, D., Laux, I., Brennan, M. and Hecht, J.R. (2015) Phase II Trial of Everolimus in Patients with Refractory Metastatic Adenocarcinoma of the Esophagus, Gastroesophageal Junction and Stomach: Possible Role for Predictive Biomarkers. Cancer Chemotherapy and Pharmacology, 76, 61-67.

https://doi.org/10.1007/s00280-015-2744-5

[19] Park, J.H., Ryu, M.H., Park, Y.S., Park, S.R., Na, Y.S., Rhoo, B.Y. and Kang, Y.K. (2015) Successful Control of Heavily Pretreated Metastatic Gastric Cancer with the mTOR Inhibitor Everolimus (RAD001) in a Patient with PIK3CA Mutation and pS6 Overexpression. BMC Cancer, 15, Article No. 119.

https://doi.org/10.1186/s12885-015-1139-7

[20] Doi, T., Muro, K., Boku, N., Yamada, Y., Nishina, T., Takiuchi, H., Komatsu, Y., Hamamoto, Y., Ohno, N., Fujita, Y., Robson, M. and Ohtsu, A. (2010) Multicenter Phase II Study of Everolimus in Patients with Previously Treated Metastatic Gastric Cancer. Journal of Clinical Oncology, 28, 1904-1910. https://doi.org/10.1200/JCO.2009.26.2923

[21] Shen, Y.C., Li, C.P., Yen, C.J., Hsu, C., Lin, Y.L., Lin, Z.Z., Chen, L.T., Su, W.C., Chao, Y., Yeh, K.H. and Cheng, A.L. (2014) Phase II Multicentered Study of Low-Dose Everolimus plus Cisplatin and Weekly 24-Hour Infusion of High-Dose 5-Fluorouracil and Leucovorin as First-Line Treatment for Patients with Advanced Gastric Cancer. Oncology, 87, 104-113. https://doi.org/10.1159/000362671

[22] Yoon, D.H., Ryu, M.H., Park, Y.S., Lee, H.J., Lee, C., Ryoo, B.Y., Lee, J.L., Chang, H.M., Kim, T.W. and Kang, Y.K. (2012) Phase II Study of Everolimus with Biomarker Exploration in Patients with Advanced Gastric Cancer Refractory to Chemotherapy Including Fluoropyrimidine and Platinum. British Journal of Cancer, 106, 1039-1044. https://doi.org/10.1038/bjc.2012.47

[23] Lee, S.J., Lee, J., Lee, J., Park, S.H., Park, J.O., Park, Y.S., Lim, H.Y., Kim, K.M., Do, I.G., Jung, S.H., Yim, D.S. and Kang, W.K. (2013) Phase II Trial of Capecitabine and Everolimus (RAD001) Combination in Refractory Gastric Cancer Patients. Investigational New Drugs, 31, 1580-1586. https://doi.org/10.1007/s10637-013-0022-0

[24] Ohtsu, A., Ajani, J.A., Bai, Y.X., Bang, Y.J., Chung, H.C., Pan, H.M., Sahmoud, T., 
Shen, L., Yeh, K.H., Chin, K., Muro, K., Kim, Y.H., Ferry, D., Tebbutt, N.C., Al-Batran, S.E., Smith, H., Costantini, C., Rizvi, S., Lebwohl, D. and Van Cutsem, E. (2013) Everolimus for Previously Treated Advanced Gastric Cancer: Results of the Randomized, Double-Blind, Phase III GRANITE-1 Study. Journal of Clinical Oncology, 31, 3935-3943. https://doi.org/10.1200/JCO.2012.48.3552

[25] Zaller, N. and Brinkley-Rubinstein, L. (2018) Incarceration, Drug Use, and Infectious Diseases: A Syndemic Still Not Addressed. The Lancet Infectious Diseases, 18, 1301-1302. https://doi.org/10.1016/S1473-3099(18)30538-3

[26] Feng, F., Jiang, Q., Cao, S., Cao, Y., Li, R., Shen, L., Zhu, H., Wang, T., Sun, L., Liang, E., Sun, H., Chai, Y., Li, X., Liu, G., Yang, R., Yang, Z., Yang, Y., Xin, S. and Li, B.A. (2018) Pregnane X Receptor Mediates Sorafenib Resistance in Advanced Hepatocellular Carcinoma. Biochimica et Biophysica Acta-General Subjects, 1862, 1017-1030. https://doi.org/10.1016/j.bbagen.2018.01.011

[27] Xie, X., Hu, H., Tong, X., Li, L., Liu, X., Chen, M., Yuan, H., Xie, X., Li, Q., Zhang, Y., Ouyang, H., Wei, M., Huang, J., Liu, P., Gan, W., Liu, Y., Xie, A., Kuai, X., Chirn, G.W., Zhou, H., Zeng, R., Hu, R., Qin, J., Meng, F.L., Wei, W., Ji, H. and Gao, D. (2018) The mTOR-S6K Pathway Links growth Signalling to DNA Damage Response by Targeting RNF168. Nature Cell Biology, 20, 320-331. https://doi.org/10.1038/s41556-017-0033-8

[28] Zhang, C.Z., Wang, X.D., Wang, H.W., Cai, Y. and Chao, L.Q. (2015) Sorafenib Inhibits Liver Cancer Growth by Decreasing mTOR, AKT, and PI3K Expression. Journal of BUON, 20, 218-222.

[29] Geissler, E.K., Schnitzbauer, A.A., Zulke, C., Lamby, P.E., Proneth, A., Duvoux, C., Burra, P., Jauch, K.W., Rentsch, M., Ganten, T.M., Schmidt, J., Settmacher, U., Heise, M., Rossi, G., Cillo, U., Kneteman, N., Adam, R., van Hoek, B., Bachellier, P., Wolf, P., Rostaing, L., Bechstein, W.O., Rizell, M., Powell, J., Hidalgo, E., Gugenheim, J., Wolters, H., Brockmann, J., Roy, A., Mutzbauer, I., Schlitt, A., Beckebaum, S., Graeb, C., Nadalin, S., Valente, U., Turrion, V.S., Jamieson, N., Scholz, T., Colledan, M., Fandrich, F., Becker, T., Soderdahl, G., Chazouilleres, O., Makisalo, H., Pageaux, G.P., Steininger, R., Soliman, T., de Jong, K.P., Pirenne, J., Margreiter, R., Pratschke, J., Pinna, A.D., Hauss, J., Schreiber, S., Strasser, S., Klempnauer, J., Troisi, R.I., Bhoori, S., Lerut, J., Bilbao, I., Klein, C.G., Konigsrainer, A., Mirza, D.F., Otto, G., Mazzaferro, V., Neuhaus, P. and Schlitt, H.J. (2016) Sirolimus Use in Liver Transplant Recipients with Hepatocellular Carcinoma: A Randomized, Multicenter, Open-Label Phase 3 Trial. Transplantation, 100, 116-125.

https://doi.org/10.1097/TP.0000000000000965

[30] Chang, L., Graham, P.H., Ni, J., Hao, J., Bucci, J., Cozzi, P.J. and Li, Y. (2015) Targeting PI3K/Akt/mTOR Signaling Pathway in the Treatment of Prostate Cancer Radioresistance. Critical Reviews in Oncology/ Hematology, 96, 507-517. https://doi.org/10.1016/j.critrevonc.2015.07.005

[31] Li, B., Yang, J., Lu, Z., Liu, B. and Liu, F. (2019) A Study on the Mechanism of Rapamycin Mediating the Sensitivity of Pancreatic Cancer Cells to Cisplatin through PI3K/AKT/mTOR Signaling Pathway. Journal of BUON, 24, 739-745.

[32] Fazio, N., Buzzoni, R., Baudin, E., Antonuzzo, L., Hubner, R.A., Lahner, H., WW, D.E.H., Raderer, M., Teule, A., Capdevila, J., Libutti, S.K., Kulke, M.H., Shah, M., Dey, D., Turri, S., Aimone, P., Massacesi, C. and Verslype, C. (2016) A Phase II Study of BEZ235 in Patients with Everolimus-Resistant, Advanced Pancreatic Neuroendocrine Tumours. Anticancer Research, 36, 713-719.

[33] Wang, H., Wang, X., Xu, L., Zhang, J. and Cao, H. (2019) A Molecular Sub-Cluster of Colon Cancer Cells with Low VDR Expression Is Sensitive to Chemotherapy, 
BRAF Inhibitors and PI3K-mTOR Inhibitors Treatment. Aging (Albany NY), 11, 8587-8603. https://doi.org/10.18632/aging.102349

[34] Liu, Y., Starr, M.D., Brady, J.C., Rushing, C., Bulusu, A., Pang, H., Honeycutt, W., Amara, A., Altomare, I., Uronis, H.E., Hurwitz, H.I. and Nixon, A.B. (2015) Biomarker Signatures Correlate with Clinical Outcome in Refractory Metastatic Colorectal Cancer Patients Receiving Bevacizumab and Everolimus. Molecular Cancer Therapeutics, 14, 1048-1056. https://doi.org/10.1158/1535-7163.MCT-14-0923-T 